Cinémas

Revue d'études cinématographiques

Journal of Film Studies

\title{
Paysage resnaisien ou variations autour de la mise en espace du temps
}

\section{Marie-Françoise Grange}

Volume 5, numéro 1-2, automne 1994

Le Temps au cinéma

URI : https://id.erudit.org/iderudit/1001010ar

DOI : https://doi.org/10.7202/1001010ar

Aller au sommaire du numéro

Éditeur(s)

Cinémas

ISSN

1181-6945 (imprimé)

1705-6500 (numérique)

Découvrir la revue

Citer cet article

Grange, M.-F. (1994). Paysage resnaisien ou variations autour de la mise en espace du temps. Cinémas, 5(1-2), 135-146. https://doi.org/10.7202/1001010ar
Résumé de l'article

À travers l'étude de l'imbrication de l'espace et du temps dans une séquence d'Hiroshima, mon amour (Alain Resnais, 1959), cette analyse s'attache à démontrer en quoi l'économie de trace définit la séquence et comment elle la constitue en une métaphore du travail mémoriel. d'utilisation que vous pouvez consulter en ligne.

https://apropos.erudit.org/fr/usagers/politique-dutilisation/ 


\title{
Paysage resnaisien ou variations autour de la mise en espace du temps
}

\section{Marie-Françoise Grange}

\begin{abstract}
RÉSUMÉ
A travers l'étude de l'imbrication de l'espace et du temps dans une séquence d'Hiroshima, mon amour (Alain Resnais, 1959), cette analyse s'attache à démontrer en quoi l'économie de trace définit la séquence et comment elle la constitue en une métaphore du travail mémoriel.

\section{ABSTRACT}

By means of a study of the interweaving of space and time in a sequence from Hiroshima, mon amour (Alain Resnais, 1959), this analysis seeks to show how the economy of traces defines the sequence and how it constitutes a metaphor for the work of memory.
\end{abstract}

Le point de départ de cet article est double. D'une part, un film, ou plutôt une séquence, extraite de Hiroshima, mon amour (Alain Resnais, 1959); d'autre part, un texte de Jacques Derrida, "Freud et la scène de l'écriture", consacré à un commentaire de "Note sur le "Bloc-notes magique" " de Freud. Une séquence, un article, deux textes, dont l'un, le dernier cité, pourrait aider à la lecture du premier.

Que Resnais soit, par excellence, le cinéaste du temps, comme beaucoup d'autres et pas des moindres ${ }^{1}$ ont su le démontrer avant moi, la séquence qui nous préoccupe ici ne pourra l'infirmer. Quant à l'article de Jacques Derrida, il est, lui 


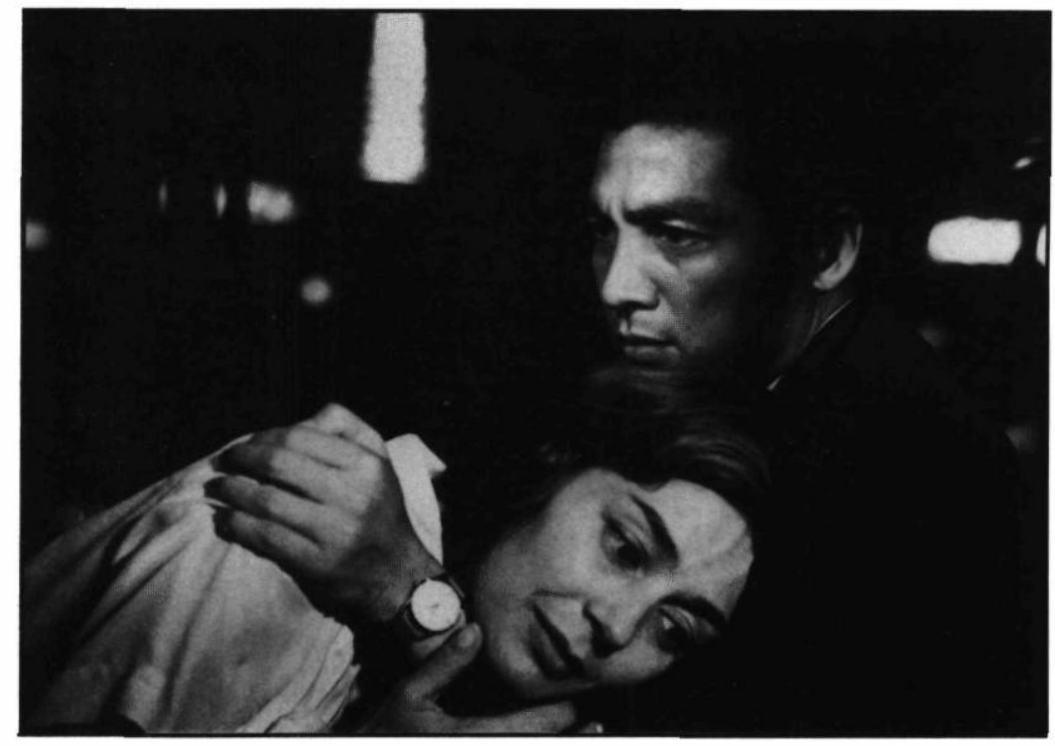

\section{Hiroshima, mon amour d'Alain Resnais (1959)}

Collection Cinémathèque québécoise

aussi axé sur la problématique temporelle et tout particulièrement sur celle mise en œuvre par Freud dans sa description du fonctionnement de l'appareil psychique.

Pourquoi cette double origine? Certes, quand il s'agit de Derrida, il vaut mieux s'armer d'un commencement qui ne pourra en être véritablement un que s'il s'enracine dans une fracture initiale. C'est reconnaître, implicitement, derrière cette boutade, que la pensée derridienne tissera la trame de ce travail. Mais encore, et plus sérieusement, il semblerait que dans cet extrait de film, caché sous un retour du passé, nous assistions au dévoilement de ce qu'est la mémoire, de ce que serait le texte de la mémoire, objet de la démonstration derridienne.

La séquence choisie nous plonge au cœur d'une discontinuité spatiale. L'héroïne, au terme de sa dernière nuit à Hiroshima, parcourt la ville sans but apparent, si ce n'est celui d'avoir à tuer le temps qu'il lui reste à passer au Japon. Suivie dans un premier moment par son amant japonais, elle déambulera, dans la suite de la séquence, seule, dans un espace tissé de deux lieux distincts: Hiroshima se mêlera alors à Nevers, lieu où elle vécut 
quatorze ans auparavant une liaison passionnelle avec un soldat allemand. Dix-neuf plans en tout vont élaborer ce va-et-vient entre deux espaces (Nevers/Hiroshima), entre deux temps (passé/présent), entre deux repères spatio-temporels qui, mis en tension par le travail filmique, perdront dans leur représentation leur valeur de stabilisation du récit.

La première caractéristique de ce passage est très certainement l'ambiguïté de l'espace ainsi construit, mais surtout l'ambiguïté quant à la prise en charge de la narration. À qui attribuer la mise en place de ce retour du passé? Qu'il y ait refus de psychologisation du flash-back, cela est évident. Ce n'est pas l'héroïne qui choisit, consciemment, de retourner à Nevers. Rien n'est fait dans ce sens-là : pas de regard perdu à l'horizon, pas d'attitude pensive, pas d'effets de ponctuation, aucun ingrédient de la construction d'un flash-back attribuable à un personnage. Pourtant, nous avons une voix off subjective qui circule du plan sept au dernier plan de la séquence. Mais, dans le texte verbal, une fois encore, pas d'appropriation de la plongée dans le passé, pas de nomination de la ville de Nevers, pas de référence précise au passé nivernais. Nous sommes là, confrontés à l'émergence d'images relatives au passé de l'héroïne sans pour autant que soit revendiqué par elle l'acte de remémoration. Cette absence de psychologisation du flash-back est le premier élément. En revanche, par l'utilisation de la voix subjective, nous pénétrons au cœur de la pensée du personnage, de son imaginaire, de son errance face à sa tentative de réappropriation discursive (verbale) des événements qu'elle a vécus. Nous pouvons reprendre ici cette affirmation de Michèle Lagny, Marie-Claire Ropars et Pierre Sorlin à propos d'un film français des années trente: "[La séquence] nous donne un savoir sur le flux mental du personnage, [elle] ne nous propose rien sur le voir du personnage" (p. 113). Impossible de nier ici le double mouvement presque contradictoire du son et de l'image. Car le texte verbal, par son inscription de signifiants verbaux, tend dans sa coprésence avec l'image à s'affirmer comme médiateur narratif du texte filmique. D'autant qu'à l'espace déconstruit font écho l'unité de la voix off et la continuité de sa présence du plan 7 au plan 19. Ce phénomène d'aspiration vers la prise en charge du récit par le texte verbal est blo- 
qué par la construction séquentielle de la bande-image. Le personnage parlant ne devient pas médiateur de perception visuelle: même si la mobilité de la caméra est parfois assujettie au déplacement du personnage, même si la contre-plongée pourrait correspondre à un regard subjectif. Le travelling, par sa régularité et sa récurrence systématique (tous les plans de la séquence sont mobiles), désanthropomorphise à la fois l'angle de prise de vue et la vision posée sur ces rues, rendues étranges par leur juxtaposition. Ce qui ne doit pas nous faire oublier les correspondances de son et d'image dont la simultanéité fait flotter indubitablement objectivité et subjectivité. Les deux systèmes cohabitent: monologue intérieur d'un côté, représentations non focalisées par un personnage de l'autre. Cela entraînera plusieurs conséquences, notamment sur les plans constitutifs du flash-back proprement dit, à savoir les plans plus spécifiquement consacrés à Nevers.

N'étant pas plus assumées par le personnage que les autres plans de la séquence, les vues de Nevers tendent à perdre leur valeur de remontée dans le passé. Cela est favorisé, voire accentué, par la nature des plans proposés. Si Nevers appartient au passé du personnage, les images relatives à cette ville n'abritent pas ou plus les événements du passé. Nevers a éliminé toute présence humaine, toute action. C'est un espace vacant, qui exhibe sa vacance, l'absence de ce qui eut lieu. Images du passé sans passé, contre-pied de tout flash-back traditionnel qui, lui, aurait présentifié, rendu le déroulement du passé. Le lieu est "offert à son degré minimal [...] c'est là mais c'est là, vide. C'est là où se montre une absence à l'œuvre" (Didi-Huberman, p. 100). Les bâtiments, les rues sont repérables dans la représentation qui nous est donnée. Mais, réceptacle d'événements perdus, car non filmés, ce visible s'emplit de son manque à voir. Nevers dévoile ce phénomène du voir "quand voir c'est perdre" (p. 100), quand scintille au cœur de la problématique du voir et son inquiétude fondatrice, et l'impossibilité d'accession à sa plénitude. C'est le statut même du flash-back qui est ici à réenvisager. Il ne s'agit plus de combler cet intervalle ouvert dans le montré. Il ne s'agit plus de reconstruire le passé afin de suturer le récit. Nous sommes confrontés à une béance, mais à une béance déjà inscrite dans le plan, dans le refus de le meubler. 
S'il n'y a plus de flash-back au sens traditionnel du terme, comment définir ces vues de Nevers? À la fois insérés dans le passé et affleurant dans le présent de l'héroïne, mais non pris en charge par elle, nous pourrions saisir ces quelques plans comme l'émergence d'un présent de Nevers n'existant que dans son passé impossible à combler. L'enveloppe présente d'un passé disparu, le lieu de la mémoire dont "le contenu restera toujours défectueux, jamais narrativisé, jamais sommé" (Didi-Huberman, p. 8), en tout cas dans la séquence analysée. L'espace est producteur d'une double temporalité (passé/ présent) ou plutôt d'un temps qui doit se penser comme unité scindée. Il n'est plus le simple cadre circonstanciel des événements - et pour cause, s'ils ne sont pas représentés —, il est devenu l'énergie génératrice d'une "différentialité temporelle \%. Le présent inclut du passé, qui lui-même inclut du présent, chacun s'éveillant à sa propre perte par l'autre. Dégagé de sa "fonction de réceptacle neutre de l'action" (p. 78) comme dirait André Gardies, il intègre à part entière la dynamique du récit. Il s'investit d'un rôle "actionnel" en déstabilisant les repères temporels de l'action. Porteur d'un vide qui s'affiche en tant que tel, il agit comme médium d'éclatement de la structure spatio-temporelle. Créateur d'une nouvelle temporalité, hybride car dédoublée, il ne peut être considéré comme un simple élément définitoire de l'action, car il agit sur le cadre définitoire de cette action dont il bloque la restitution.

L'espace déclenche un procès. Il interfere sur le "circonstant". Il met en mouvement le temps de référence de l'action et cela d'une façon extrêmement intéressante. L'opération ne consiste pas à bouger sur l'axe temporel, à circuler du passé au présent et vice versa, mais à insuffler du passé dans le présent, du présent dans le passé, à grever le point de localisation temporelle, à l'espacer de lui-même: Nevers est à concevoir comme le présent d'un passé perdu, mais également comme un passé qui entraîne dans sa perte le présent. C'est l'espace d'une mémoire sans remémoration dans laquelle il n'y a plus de souvenirs, un espace vacant soumis simplement à du temps qui ne se définit plus ni comme passé ni comme présent.

On voit combien, dans les images de flash-back, la structure est complexe. Et cette complexité va glisser sur l'ensemble de la 
séquence qui n'est pas uniquement composée, je le rappelle, de plans sur Nevers.

Le flash-back est fractionné par les vues d'Hiroshima habitées pour certaines par l'héroïne marchant au hasard dans les rues. Le montage construit une ville imaginaire à partir de deux lieux distincts qui ne masquent pas leur spécificité, au contraire.

Si Nevers étale, par ses bâtiments vieille France, son aspect de ville française provinciale, Hiroshima, quant à elle, souligne, par ses enseignes lumineuses écrites en japonais, qu'ici, "ça ne s'arrête jamais". L'architecture des deux villes s'oppose, mais également le moment où elles furent filmées: le petit matin de Nevers contraste avec la nuit d' Hiroshima. La différence des lieux est inscrite dans la représentation. Ce pourrait même être elle qui soit filmée par le refus de la bande-image de fondre l'hétérogénéité des lieux. Le composite de l'espace, sa différentialité devient l'objet de la séquence. Quant à la structure temporelle, elle est entraînée dans ce jeu spatial où le lieu morcelé se veut pourtant unifié par les mouvements d'appareil omniprésents. Le passé de Nevers, monté bout à bout avec le présent d'Hiroshima, ce passé qui déjà est à concevoir comme débouté de sa fixité, est mis en relation étroite avec le présent qui cherche à se distinguer face à ce passé déstabilisé. Par le retour incessant sur le personnage, il semblerait qu'il y ait volonté de se référer à un repère temporel, celui de l'actuel, du maintenant, du présent. Mais la déconstruction spatiale intervenant dans la construction même de cet espace hétérogène (fait à la fois de Nevers et d'Hiroshima) interfere sur la détermination précise du repère en question. De quel présent s'agit-il? Certains plans répondront le présent de l'héroïne à Hiroshima, d'autres, embarqués dans le parcours sans fin d'une caméra se contentant de filmer des lieux vidés, rapprochant sans les confondre Hiroshima de Nevers, ne permettront pas de réponse aussi aisée. Hiroshima face à Nevers, mais mêlée à Nevers, fondue dans Nevers. Hiroshima différente de Nevers, mais intégrée dans Nevers et réciproquement. Hiroshima se définira, elle aussi, par une étrange temporalité.

Nevers est le lieu de la mémoire, avons-nous dit plus haut, mais d'une mémoire sans souvenir. Ce n'est là qu'espace présent désignant un passé non formalisé dans des événements. Une 
sorte d'enveloppe vide, mais constituée par ce vide qu'elle inclut. Les deux marchent ensemble et, qui plus est, ne peuvent, dans ce cas-là, se penser ni comme dissociés ni comme dissociables. Le présent de Nevers est support du passé de Nevers, il n'est rien sans ce passé. Le présent se pose comme oubli du passé, mais constitué par cet oubli qu'il est du passé. Le présent est trace du passé. Quant au passé, il n'existe que dans le présent qui le fait remonter à la surface. Ce passé est au présent. Mais en étant au présent, il devient la trace du présent. Il est le présent sans l'être vraiment, il en porte seulement l'empreinte. Ils sont tous deux dans un rapport différentiel au sens derridien du terme. D'une part, ils sont non semblables (le Nevers du présent n'est pas le Nevers du passé), d'autre part, ils different dans le temps. Non seulement ils sont distincts l'un de l'autre (même s'ils sont indissociables) mais encore, l'un intégrant l'autre, differe l'autre, ils se different d'eux-mêmes. Car que devient un passé qui n'est plus à concevoir comme un ancien présent, mais comme constitutif du présent? Que devient ce présent qui est espacé de lui-même par le fait qu'il doit se définir avec ce passé qui le constitue? Derrida affirmera qu'une telle conception temporelle oblige à penser "une autre stratification du temps" (1967a, p. 325). "Chaque élément dit "présent", apparaissant sur la scène de la présence, se rapporte à autre chose que luimême, gardant en lui la marque de l'élément passé et se laissant déjà creuser par la marque de son futur. [...] Il faut qu'un intervalle le sépare de ce qui n'est pas lui pour qu'il soit lui-même, mais cet intervalle qui le constitue en présent doit aussi du même coup diviser le présent en lui-même [...]" (1972, p. 13). Il me semble que Nevers, saisie dans le type de flash-back analysé, relève plus de cette conception temporelle que d'une conception classique, linéaire du temps.

Ce travail qui s'effectue sur le temps propre de Nevers se reporte sur celui d'Hiroshima et cela, malgré les tentatives faites par les plans consacrés au personnage marchant dans les rues pour définir un présent de référence. En quoi il y a-t-il transfert? C'est le maintenant d'Hiroshima qui fait naître l'autrefois de Nevers, mais un autrefois qui, comme nous l'avons vu, est pour le moins ambigu, puisque c'est un autrefois non repré- 
senté, la trace de cet autrefois. C'est à Hiroshima que surgit Nevers, l'absence de ce qui y fut vécu. Nevers, insufflée dans Hiroshima, fait d'Hiroshima le retour de Nevers, le retour de la trace de Nevers, le présent de cet autrefois vidé, la trace de cette trace. La mémoire d'Hiroshima se structure, à son tour, de couches différentielles. Incluant Nevers, elle se tourne vers le passé de l'héroïne, ce passé absent qui n'existe que comme empreinte temporelle. Elle définit le présent comme reprise du passé (constitué, par ailleurs, comme trace) et le passé comme reprise du présent. Se promener à Hiroshima, c'est se promener à Nevers. Les deux propositions sont équivalentes. Le traitement représentationnel des deux villes ( $c f$. les incessants mouvements d'appareil) ne nous démentira pas. Hiroshima répète Nevers. Et, en répétant Nevers, se conçoit comme la trace de Nevers. Mais si Hiroshima est un autre Nevers, la proposition se retourne également: Nevers est la trace d'Hiroshima, elle est un autre Hiroshima, la trace de sa perte à venir. Et Nevers, à son tour, differe Hiroshima. Les deux villes et les événements qui s'y rattachent perdent la plénitude de leur présence. Chacune se rapporte à autre chose, n'existe que par cette autre chose (les événements de l'autre ville) à laquelle elle se réfere.

La première conséquence d'un tel dispositif est très certainement le flottement du flash-back. Car ce flash-back qui, déjà, n'en est pas vraiment un ( $c f$. sa non-psychologisation) se construit en fait sur deux présents, celui de Nevers (emplis de son propre passé) et celui d'Hiroshima incluant le passé/ présent de Nevers. La structure même de la séquence, l'ambiguïté de sa construction spatiale, fait en sorte qu'elle relève davantage d'un syntagme alterné que d'un flash-back.

Les plans de Nevers et ceux d'Hiroshima se succédant, alternant, définissent leurs espaces comme simultanés. Que les événements qui s'y sont déroulés ne soient pas, eux, simultanés ne pose pas de problème particulier puisqu'ils ne sont pas (directement) représentés. Et pourtant, les deux villes montrées valent toute deux pour chacune des histoires d'amour qui s'y sont déroulées. Quant à l'héroïne que nous voyons parfois déambuler dans Hiroshima, elle est saisie dans la simultanéité des deux espaces, dans la simultanéité des deux histoires vidées de leur présence. 
Elle est confrontée au rapport différentiel de ces deux moments vécus. En ce sens, l'espace ainsi élaboré de deux lieux distincts s'ouvre sur le mouvement dans lequel est prise la mémoire du sujet. La Française, dans les rues d'Hiroshima-Nevers, ne se souvient de rien, elle ne fait que subir l'emprise temporelle, différentielle de sa mémoire. Sa mémoire n'est plus le cumul d'événements qui eurent lieu à une époque donnée, elle ne se structure plus à partir d'un moment originaire. Cela ne part ni de Nevers ni d'Hiroshima, mais de cette double entité qui n'en fait qu'une. Et Hiroshima, pieds et poings liés à Nevers, projette un futur aussi dévasté que celui de ce passé / présent. Hiroshima, à l'image de Nevers, ne sera rien, ne sera que ce flottement de la trace qui travaille à l'effacement de tout événement quelle qu'en soit son importance. Le texte verbal se fait écho d'une telle évidence: "Nous pleurerons le jour défunt avec conscience et bonne volonté. Nous n'aurons plus rien d'autre à faire que de pleurer le jour défunt. Du temps passera, du temps seulement, et du temps va venir, du temps viendra où nous ne saurons plus du tout nommer ce qui nous unira" (plans 18 et 19). Mais c'est au principe même de la structure temporelle que doit être rattaché ce travail de la trace. Car si aucun point ne se fixe dans le temps comme repère d'une conscience tout à sa maîtrise et présence à soi, cela est tributaire, d'une part, de l'effondrement de la linéarité de l'axe temporel, d'autre part, de la nécessité de repenser le repère temporel selon la problématique de la trace, effacée, effaçante. "La trace est l'effacement de soi, de sa propre présence, elle est constituée par la menace ou l'angoisse de sa disparition irrémédiable, de la disparition de sa disparition" (Derrida, 1967a, p. 339). Or, Hiroshima est le lieu de la perte de Nevers, là où ce qui reste de Nevers, sa trace, est soumis au processus d'effacement. L'amour d'Hiroshima s'écrit sur la relation avec l'Allemand. Ce faisant, il efface cette relation antérieure. Mais en effaçant l'autre, il porte en germe sa propre mort, car il nous dit n'être rien sans l'Allemand et pourtant, il en détruit l'histoire. Hiroshima est bien le lieu où naît l'angoisse de la "disparition irrémédiable " de la trace, de "la disparition de sa disparition ".

C'est dans la mise en scène de l'espace que se met en forme cette temporalité de la trace. Elle est son devenir, son devenant, 
le processus de sa formalisation. La structuration de l'espace ébranle le point de référence temporel de l'événement et lui confere, de l'intérieur, son impossibilité originaire. Plus de départ, plus de commencement, plus d'origine, l'événement ne s'inscrit plus à un moment donné de la chaîne et à un seul endroit déterminé, il est saisi dans le flux de sa temporalisation, cette temporalisation qui implique l'effacement. C'est dire que l'espace n'arrive pas après coup, sur une structure temporelle déjà élaborée, mais qu'il est l'élaboration de cette structure temporelle. Dans cet espace disjonctif et conjoint de la séquence advient la texture de ce temps en train de se faire. S'inscrivant dans la problématique de la différance au sens derridien, le lieu Nevers-Hiroshima écarte l'événement de son origine pour le relier à l'autre événement qui le fait exister en l'effaçant. Dans l'expression "relier à ", nous renvoyons à ce travail de temporalisation, à savoir, la mise en relation temporelle de l'événement à son autre lui-même, le parcours du temps qui le sépare de luimême, son espacement interne. Si l'espace séquentiel ainsi décrit est la scène qui engendre les relations de trace à trace, où le personnage-sujet se perd dans le flux temporel, ne pourrions-nous pas alors considérer cet espace comme lieu mémoriel, c'est-àdire comme lieu où s'écrit le mouvement différentiel de la mémoire? C'est ni plus ni moins à la structure de la mémoire mise en valeur par Jacques Derrida dans sa lecture de "La Note sur le "Bloc-notes magique" " de Freud que nous nous référons.

Que nous dit Derrida dans son analyse du texte freudien? Sans vouloir entrer dans les détails, nous retiendrons que pour Freud " la mémoire n'est [...] pas une propriété du psychisme parmi d'autres, elle est l'essence même du psychisme " (1967a, p. 299). Décrire le fonctionnement de l'appareil psychique revient donc à (d')écrire celui de la mémoire. Ensuite, s'attachant particulièrement au parcours de la trace, Derrida relève comment, dans la conception freudienne de l'appareil psychique, se déconstruit tout présent et donc tout passé ayant été présent. Repoussant la notion d'atemporalité de l'inconscient, Derrida affirme qu'il est bien là question de temps, même si celui-ci doit être conceptualisé à partir d'un espacement interne qui change la face de toute temporalité classiquement définie. Que 
devons-nous entendre par ce terme d'espacement? Ce que Derrida définit lui-même comme mise en espace du temps et mise en temps de l'espace. Plus que l'imbrication indéfectible de ces deux éléments, il faut voir dans ce "devenir-espace du temps » et ce "devenir-temps de l'espace" (1967b, p. 99) le trajet de la trace qui construit, en se développant, son propre espace. Premièrement, l'itinérance temporelle nécessite un, son déroulement spatial; deuxièmement, en paraphrasant Derrida, nous pouvons dire que la trace produit l'espace et ne se contente pas de parcourir un espace déjà existant ${ }^{2}$. Le temps prend forme en parcourant sa distance interne. Quant à l'espace, il se dessine dans ce trajet temporel. L'homologie est à noter entre notre séquence et la conception ainsi définie du travail mémoriel.

L'héroïne erre dans une ville inconnue dont la topographie se forge au fur et à mesure de la déambulation d'une caméra itinérante et d'un montage défiant les limites d'un territoire circonscrit. Les travellings latéraux, avant, arrière, agrémentés de quelques panoramiques, ouvrent un espace en cours de formation, pénètrent le lieu qui se temporalise dans cette effraction de l'appareil, dans le "frayage" que le mouvement opère à travers les rues désertes. La caméra trace l'espace et le chemin de la trace. Elle inscrit cette trace dans une durée qui n'est pas seulement celle du plan mais celle d'un trajet en train de s'accomplir, un trajet profilant la route de son cheminement. Le montage, lui, met en relation les différentes couches et autorise la traversée des zones disjointes et communicantes à la fois. Et nous retrouvons là les principes à la base du système psychique tel que Derrida le lit dans le "bloc magique freudien": le frayage à travers les strates, l'effraction permettant le contact entre les différentes couches psychiques, l'inscription toujours redoublée et jamais originaire de la trace écartelée par le trajet qui la constitue.

Tout comme le "bloc magique», métaphore choisie par Freud pour rendre compte du travail mémoriel, la séquence nous joue la scène de l'écriture, cette écriture dont "[...] la condition [est] qu'il n'y ait ni contact permanent ni une rupture absolue entre les couches" (Derrida, 1967a, p. 335). Juste un passage, une trajectoire à travers des lieux si proches et si distants, dans lequel circule l'énergie frayante et compulsive d'une mémoire sans 
sujet, d'une mémoire sans origine qui entraîne dans son mouvement différentiel la Française perdue dans les rues d'HiroshimaNevers.

\section{Université de Saint-Étienne}

\section{NOTES}

1 Je pense notamment à Gilles Deleuze.

2 «[...] travail itinérant de la trace, produisant et non parcourant sa route, de la trace qui trace, de la trace qui se fraye elle-même son chemin " ("Freud et la scène de l'écriture ", L'Écriture et la différence, Paris: Seuil, 1967a, p. 317).

\section{OUVRAGES CITÉS}

Derrida, Jacques. "La différance ", Marges. De la philosophie. Paris: Minuit, 1972.

Derrida, Jacques. "Freud et la scène de l'écriture", L'Écriture et la différence. Paris: Seuil, 1967a.

Derrida, Jacques. De la grammatologie. Paris: Minuit, $1967 \mathrm{~b}$.

Didi-Huberman, Georges. Ce que nous voyons, ce qui nous regarde. Paris: Minuit, 1992.

Freud, Sigmund. "Note sur le "Bloc-notes magique" ", Résultats, idées, problèmes 1921-1938, tome 2. Paris : P.U.F., 1985.

Gardies, André. Le Récit filmique. Paris: Hachette, 1993.

Lagny, Michèle, Ropars, Marie-Claire et Sorlin, Pierre. "Le récit saisi par le film ". Hors Cadre, $\mathrm{n}^{\circ} 2$ (1984). 